\title{
Best of Milan 2017-ERS Lung Science Conference session "Lung tissue repair and remodeling in chronic lung diseases: mechanisms and therapeutic approaches"
}

\author{
Sabine Bartel ${ }^{1}$, Catherine M. Greene ${ }^{2}$ \\ ${ }^{1}$ Early Life Origins of Chronic Lung Disease, Research Center Borstel, Leibniz Center for Medicine and Biosciences, member of the German Center \\ for Lung Research (DZL) and Airway Research Center North (ARCN), Borstel, Germany; ${ }^{2}$ Department of Microbiology, Royal College of Surgeons \\ in Ireland, Education and Research Centre, Beaumont Hospital, Dublin, Ireland \\ Correspondence to: Dr. Sabine Bartel. Leibniz Center for Medicine \& Biosciences, Borstel, Germany. Email: sbartel@fz-borstel.de.
}

Submitted Aug 23, 2017. Accepted for publication Sep 29, 2017.

doi: $10.21037 /$ jtd.2017.11.40

View this article at: http://dx.doi.org/10.21037/jtd.2017.11.40

During the Annual Congress of the European Respiratory Society in Milan 2017, a variety of exciting developments in basic and clinical respiratory research was presented and discussed. One of the main foci of the conference was novel developments in chronic lung diseases, such as chronic obstructive pulmonary disease (COPD). As the incidence of COPD (and other chronic lung diseases) has markedly increased over the last decades and is predicted to continue increasing in the following years (1), there is a huge clinical need to develop novel therapeutic or preventative strategies. COPD is associated with marked injury and remodeling of the airways and alveoli as well as the surrounding lung tissue without adequate repair. However the underlying molecular events leading to the development of this disease are at present not fully understood. This review will summarize some of the latest findings in the field of lung tissue repair and remodeling, including potential opportunities for novel therapies, which was the central topic of one of the scientific symposia in Milan.

\section{Wingless/integrase-1 (WNT) signaling in lung repair and remodeling}

The WNT signaling pathway has classically been described for its essential role in organ development, however alterations thereof have also been associated with the pathogenesis of diseases such as COPD (2-4). Upon binding to various transmembrane receptors, the different WNT ligands can induce two different responses: either activating the $\beta$-catenin dependent (canonical) or $\beta$-catenin independent (non-canonical) signaling pathways [reviewed in (5)]. In a recent study, Baarsma et al. observed a transition from canonical to noncanonical WNT signaling during the development of COPD (6). Along this line, noncanonical signaling was induced as a result of increased expression of the ligand WNT-5A in lung tissue of two different mouse models for emphysema (chronic cigarette smoke exposure and elastase treatment) and human COPD patients (6). Further, they identified primary human lung fibroblasts (phLF) as main producer of WNT-5A, which was increased in fibroblasts obtained from COPD patients and could be increased in vitro by TGF- $\beta$ and cigarette smoke extract (6). The secreted WNT-5A was able to negatively regulate canonical ( $\beta$-catenin dependent) WNT signaling in vitro in alveolar epithelial cell lines, leading to an impaired wound healing capacity (6). Additionally, transgenic, lung-specific overexpression of WNT-5A aggravated the development of elastase-induced emphysema, while prophylactic neutralization of WNT-5A by antibodies ameliorated tissue damage and lung function decline after elastase treatment (6). Of note, treatment with a WNT-5A neutralizing antibody also therapeutically improved the outcome of established elastase-induced emphysema (6).

Thereby, this study provides critical proof that WNT-5A signaling is involved in the development of emphysema, potentially by inhibiting the $\beta$-catenin-driven repair pathways in alveolar epithelial cells. Thus, targeting WNT-5A should be further investigated as a potential novel therapy for COPD. 


\section{The role of basal cells in aberrant epithelial repair}

The human airway epithelium is, to a certain extent, able to repair itself upon injury or infection. This process is partly mediated by airway basal cells, which are considered as stem or progenitor cells for the airways (7). Thus, an aberrant epithelial repair might be due to an underlying defect in the basal cell population.

To further investigate this, a recent study by Yang and colleagues analyzed the transcriptomic profiles of small airway epithelial cells, obtained by bronchoscopic brushing of $10^{\text {th }}-12^{\text {th }}$ generation bronchi (8). By comparing the expression profiles of large and small airway epithelium (SAE), the authors were able to develop a proximal-to-distal airway gene expression signature (8). Of note, the differences in expression were not simply due to a difference in cellular composition, but rather a distinct signature respective of the environment of e.g., ciliated cells (8). In SAE of smokers with or without COPD, the distal signature was decreased, while the proximal genes were upregulated-an observation which the authors termed 'distal-to-proximal repatterning' (8). Further, they demonstrated that the degree of "proximalization" correlated with lung function $\left(\mathrm{FEV}_{1} / \mathrm{FVC}\right.$ ratio), indicating functional consequences of those genomic alterations (8). A pathway analysis identified the EGF/EGFR signaling pathway as main regulator of the proximalization (P)-signature genes, which were also confirmed to be functionally relevant in in vitro exposures of primary airway epithelial cells to cigarette smoke extract (8). As these transcriptomic alterations were found in regions without histological abnormalities, the authors speculated that these events occur very early in the development of COPD (8).

This study gives important insight in early mechanisms and effects of cigarette smoke on airway epithelial cells. It also indicates that the EGFR signaling pathway might be another potential therapeutic target for COPD.

\section{Identifying remodeling and therapeutic targets using a small airway-on-a-chip}

As mentioned above, primary airway epithelial cells can be cultured in vitro and differentiated at the air-liquid interface to a pseudostratified epithelium with the same cellular composition as in the lung (9). While being advantageous over regular submerged cultures of cell lines, these cultures however still do not represent the real lung microenvironment due to their purely epithelial composition.

In order to circumvent this limitation, Benam and colleagues have developed a very elegant small airway-on-a-chip.
Hereby, the differentiated mucociliary bronchiolar epithelium is underlined by a microvascular endothelium also featuring fluid flow to model the lung pathophysiology in vitro (10). This microfluidic device was prepared using poly(dimethylsiloxane) (PDMS), while the upper 'air channel' ( $1 \mathrm{~mm}$ high $\times 1 \mathrm{~mm}$ wide) was separated by a $0.4-\mu \mathrm{m}$ porous polyester membrane from the lower 'blood channel' $(0.2 \mathrm{~mm}$ high $\times 1 \mathrm{~mm}$ wide). The primary airway epithelial cells were seeded on top of the type I collagen-coated membrane and differentiated for 3 to 5 weeks under air-liquid interface conditions (10). Afterwards, primary human lung microvascular endothelial cells were applied to the opposite side of the membrane. Further, using microfluidics a continuous flow of medium was supplied to the lower 'blood channel' at a rate of $60 \mu \mathrm{L} \cdot \mathrm{h}^{-1}$, mimicking pulmonary blood flow (10). Thus, this airway-on-a-chip is suitable to investigate epithelial differentiation and function by enabling immunofluorescence, electron microscopy, ciliary beat frequency and mucociliary transport measurements (10). Additionally, complex biological processes such as neutrophil recruitment, rolling and adhesion can be investigated by introducing primary human neutrophils to the 'blood channel' (10). Furthermore, the system allows researchers to measure cytokine secretion and lately also trans-epithelial electrical resistance (TEER) (11) in real-time after application of stimuli such as IL-13 treatment to mimic asthma (10), cigarette smoke exposure (12) or viral and bacterial infections (10).

Thus, this small airway-on-a-chip is of tremendous potential for future research to translate findings from mouse models into humans and to test novel therapeutic strategies for e.g., asthma, COPD or infections.

\section{Epithelial stem cells and airway tissue engineering}

As discussed above, airway basal cells are considered as stem/ progenitor cells in the lung, thus it is an intriguing concept to try to use them to repair injured lung tissue or even to manufacture airways for regenerative transplantation. Along this line, tissue-engineered tracheas have been transplanted since 2008, while the seeding of scaffolds with autologous epithelial cells has been reported to be beneficial $(13,14)$. However, for urgent re-cellularization of bioengineered scaffolds there is often insufficient time for isolation and expansion of autologous airway epithelial cells to obtain the required cell numbers by standard methods. Thus, Butler and colleagues have developed a novel and faster method to obtain larger numbers of functional airway basal cells (15). Based on existing work in the stem cell field (16), they 
compared a co-culture with mitotically inactivated 3T3-J2 fibroblast feeder cells in the presence of Rho-associated protein kinase (ROCK) inhibition (inhibitor Y27632) $(3 \mathrm{~T} 3+\mathrm{Y})$, to the classical expansion in bronchial epithelial growth medium (BEGM) (15). Basal cells cultured under $3 \mathrm{~T} 3+\mathrm{Y}$ conditions proliferated faster than those grown in BEGM, retained their normal karyotype after 6 weeks in culture, were able to differentiate into a pseudostratified epithelium at the air-liquid interface, as well as to form tracheospheres, also in late passages (15). In a xenogeneic engraftment model, where cells could engraft for $6 \mathrm{~h}$ on scaffolds before they were subcutaneously implanted in NOD SCID gamma mice, the authors could show that the human cells restored an epithelial barrier around the tracheal graft in vivo after 5 weeks (15). Of note, this method was also able to functionally expand airway basal cells from cryopreserved patient samples (17).

In conclusion, the co-culture with 3T3-J2 feeder cells with ROCK inhibition almost doubled the culture success of basal cells and generated large cell numbers and thus offers great potential for tissue engineering approaches in regenerative medicine.

\section{Acknowledgements}

None.

\section{Footnote}

Conflicts of Interest: The authors have no conflicts of interest to declare.

\section{References}

1. European Lung White Book. The burden of lung disease. Introduction: the global perspective. (2014). Available online: https://www.erswhitebook.org/chapters/theburden-of-lung-disease/

2. Kneidinger N, Yildirim AÖ, Callegari J, et al. Activation of the $\mathrm{WNT} / \beta$-catenin pathway attenuates experimental emphysema. Am J Respir Crit Care Med 2011;183:723-33.

3. Heijink IH, de Bruin HG, van den Berge M, et al. Role of aberrant WNT signalling in the airway epithelial response to cigarette smoke in chronic obstructive pulmonary disease. Thorax 2013;68:709-16.

4. Uhl FE, Vierkotten S, Wagner DE, et al. Preclinical validation and imaging of Wnt-induced repair in human 3D lung tissue cultures. Eur Respir J 2015;46:1150-66.

5. Baarsma HA, Königshoff $M$. 'WNT-er is coming': WNT signalling in chronic lung diseases. Thorax 2017;72:746-59.

6. Baarsma HA, Skronska-Wasek W, Mutze K, et al. Noncanonical WNT-5A signaling impairs endogenous lung repair in COPD. J Exp Med 2017;214:143-63.

7. Hogan BL, Barkauskas CE, Chapman HA, et al. Repair and regeneration of the respiratory system: complexity, plasticity, and mechanisms of lung stem cell function. Cell Stem Cell 2014;15:123-38.

8. Yang J, Zuo WL, Fukui T, et al. Smoking-Dependent Distal-to-Proximal Repatterning of the Adult Human Small Airway Epithelium. Am J Respir Crit Care Med 2017;196:340-52.

9. Fulcher ML, Gabriel S, Burns KA, et al. Welldifferentiated human airway epithelial cell cultures. Methods Mol Med 2005;107:183-206.

10. Benam KH, Villenave R, Lucchesi C, et al. Small airwayon-a-chip enables analysis of human lung inflammation and drug responses in vitro. Nat Methods 2016;13:151-7.

11. Henry OYF, Villenave R, Cronce MJ, et al. Organson-chips with integrated electrodes for trans-epithelial electrical resistance (TEER) measurements of human epithelial barrier function. Lab Chip 2017;17:2264-71.

12. Benam KH, Novak R, Nawroth J, et al. MatchedComparative Modeling of Normal and Diseased Human Airway Responses Using a Microengineered Breathing Lung Chip. Cell Syst 2016;3:456-66.e4.

13. Macchiarini P, Jungebluth $\mathrm{P}, \mathrm{Go} T$, et al. Clinical transplantation of a tissue-engineered airway. Lancet 2008;372:2023-30.

14. Elliott MJ, De Coppi P, Speggiorin S, et al. Stem-cellbased, tissue engineered tracheal replacement in a child: a 2-year follow-up study. Lancet 2012;380:994-1000.

15. Butler CR, Hynds RE, Gowers KH, et al. Rapid Expansion of Human Epithelial Stem Cells Suitable for Airway Tissue Engineering. Am J Respir Crit Care Med 2016;194:156-68.

16. Liu X, Ory V, Chapman S, et al. ROCK inhibitor and feeder cells induce the conditional reprogramming of epithelial cells. Am J Pathol 2012;180:599-607.

17. Gowers KHC, Hynds RE, Thakrar RM, et al. Optimized isolation and expansion of human airway epithelial basal cells from endobronchial biopsy samples. J Tissue Eng Regen Med 2017. [Epub ahead of print].

Cite this article as: Bartel S, Greene CM. Best of Milan 2017-ERS Lung Science Conference session "Lung tissue repair and remodeling in chronic lung diseases: mechanisms and therapeutic approaches". J Thorac Dis 2017;9(Suppl 16):S1541S1543. doi: 10.21037/jtd.2017.11.40 